\section{JTI}

JOURNAL OF

TRAUMA AND INJURY

\title{
Aortoesophageal Fistula after Thoracic Endovascular Aortic Repair for Blunt Thoracic Aortic Injury
}

\author{
Masakazu Nitta, M.D., Ph.D., Taro Tamakawa, M.D., Natsuo Kamimura, M.D., \\ Tadayuki Honda, M.D., Ph.D., Hiroshi Endoh, M.D., Ph.D. \\ Advanced Emergency and Critical Care Center, Niigata University Medical and Dental \\ Hospital, Niigata, Japan
}

Received: July 26, 2019

Revised: August 25, 2019

Accepted: August 25, 2019

\section{Correspondence to}

Masakazu Nitta, M.D., Ph.D.

Advanced Emergency and Critical Care Center, Niigata University Medical and

Dental Hospital, 754 Asahimachi-Dori, Chuo-ku, Niigata 951-8520, Japan

Tel: $+81-25-227-2338$

Fax: +81-25-227-0791

E-mail:masakazunitta@gmail.com
Although thoracic endovascular aortic repair (TEVAR) has grown to become the standard of care to treat blunt thoracic aortic injury (BTAI), the long-term effects of TEVAR are still unclear. We here present a 72-year-old man with BTAI due to a traffic accident. He successfully underwent TEVAR and was transferred to another rehabilitation hospital 2 months after the accident. However, 1 month later, he underwent gastroscopy with fever and hematemesis and was diagnosed with aorto-esophageal fistula (AEF). After being re-transferred to Niigata University Medical and Dental Hospital, we tried to convince him to undergo surgical treatment, but he strongly refused. He received palliative care and died due to rupture of the aortic pseudoaneurysm 3 days after the hospital transfer. Fatal complications like AEF may occur after TEVAR, so clinicians need to carefully follow patients who underwent TEVAR.

Keywords: Esophageal fistula; Aortic rupture; Frailty; Endovascular procedures

\section{INTRODUCTION}

Since thoracic endovascular aortic repair (TEVAR) has taken the place of surgical repair and grown to become the standard of care to treat blunt thoracic aortic injury (BTAI), the mortality of BTAI is decreasing [1-3]. But the long-term effects of TEVAR are still unclear. We here present a case that, although a patient suffering from BTAI successfully underwent TEVAR, he eventually died due to rupture of the aortic pseudoaneurysm related to an aortoesophageal fistula (AEF). 


\section{CASE REPORT}

A 72-year-old man was hit by a car and initially brought to another hospital with hemorrhagic shock. After BTAI with hemothorax and free air in the abdomen were diagnosed by a computerized tomography (CT) scan, he was transferred to emergency department of Niigata University Medical and Dental Hospital (Fig. 1).

On arrival, hemorrhagic shock had been prolonged (blood pressure of $47 / 36 \mathrm{mmHg}$ and a heart rate of $67 \mathrm{bpm}$ ). In addition, hypothermia (temperature of $32^{\circ} \mathrm{C}$ ), acidosis ( $\mathrm{pH} 7.14$ ), and coagulopathy (international normalized ratio of 1.59 and serum fibrinogen of $75 \mathrm{mg} / \mathrm{dL}$ ), known as the lethal triad, had already developed. According to the CT scan, his injury severity score was $38\left(2^{2}\right.$ [facial fractures $]+5^{2}$ [BTAI] $+3^{2}$ [intestinal injuries] $)$ and the probability of survival was $9 \%$. Active bleeding through the right chest tube, which was placed at the previous hospital, had continued and the amount of bleeding had been over 2,200 $\mathrm{mL}$ on arrival (about 4 hours after the accident). Because we recognized that BTAI was the cause of bleeding that is difficult to stop by emergency thoracotomy, we thought that thoracotomy at that time would cause cardiac arrest due to a reduction of intrathoracic pressure. Thus, we clamped the tube expecting hemostasis with the rise of intrathoracic pressure and vital signs were slightly ameliorated (blood pressure of $84 / 55 \mathrm{mmHg}$ and a heart rate of $66 \mathrm{bpm})$.
After we applied therapeutic strategies of administration of fibrinogen concentrate, permissive hypotension, and active warming, the lethal triad started to resolve and his vital signs improved. He was then transferred to the operating room and successfully underwent TEVAR. Upon completion of TEVAR, the lethal triad had almost disappeared. He subsequently underwent a laparotomy. Eventually, two lacerations of the small bowel were found and repaired. After these procedures, he was admitted to the intensive care unit in a hemodynamically stable state. On day 4, he recovered consciousness. Thereafter, he went through rehabilitation programs. The follow-up chest X-ray and CT scan on day 30 did not show any problems related to TEVAR (Fig. 2). He was transferred to another rehabilitation hospital on day 60 .

However, 1 month after the hospital transfer, he unexpectedly had a fever and hematemesis. He underwent gastroscopy, which revealed an AEF (Fig. 3). He was re-transferred to Niigata University Medical and Dental Hospital and a CT scan showed mediastinitis with an aortic pseudoaneurysm (Fig. 4). We tried to convince him to undergo re-TEVAR and surgical treatment of the esophagus, but he strongly refused such treatments. He received palliative care and died due to rupture of the aortic pseudoaneurysm related to the AEF 3 days after the hospital transfer.
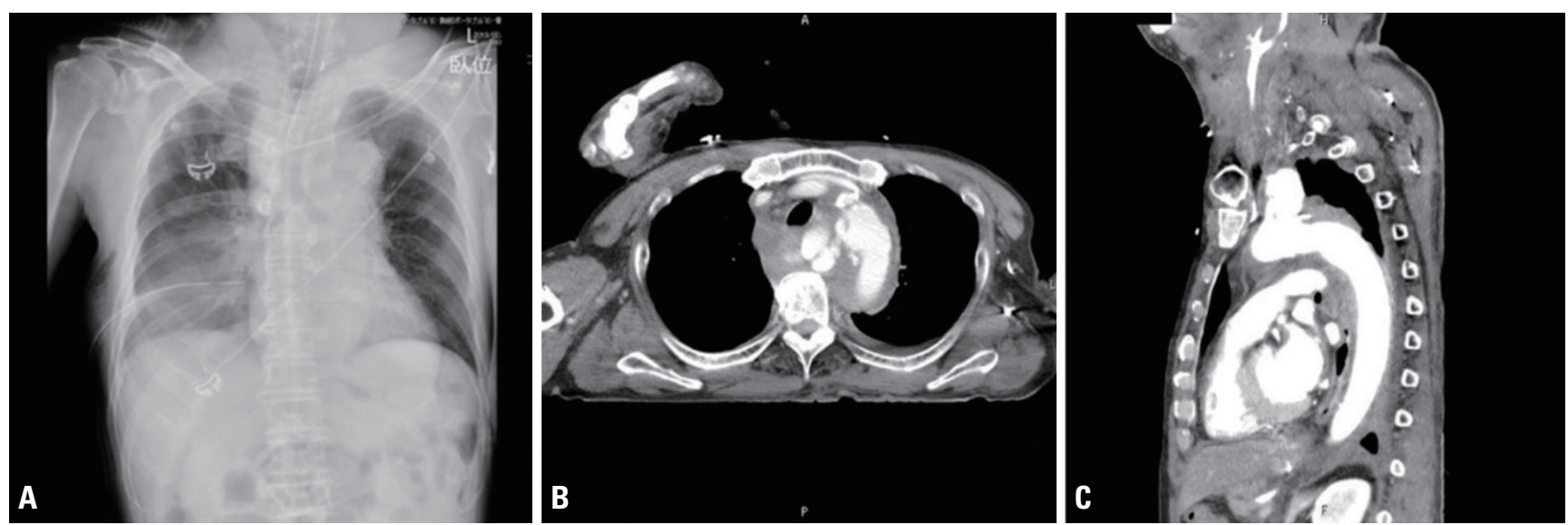

Fig. 1. A chest $X$-ray $(A)$ and a chest $C T$ scan with contrast $(B, C)$ on arrival. He had already been intubated at the previous hospital, and a chest tube had also been placed in the right cavity ( $A$ ). The $C T$ scan revealed a ruptured pseudoaneurysm of the isthmus (B, C). CT: computerized tomography. 


\section{JTI}
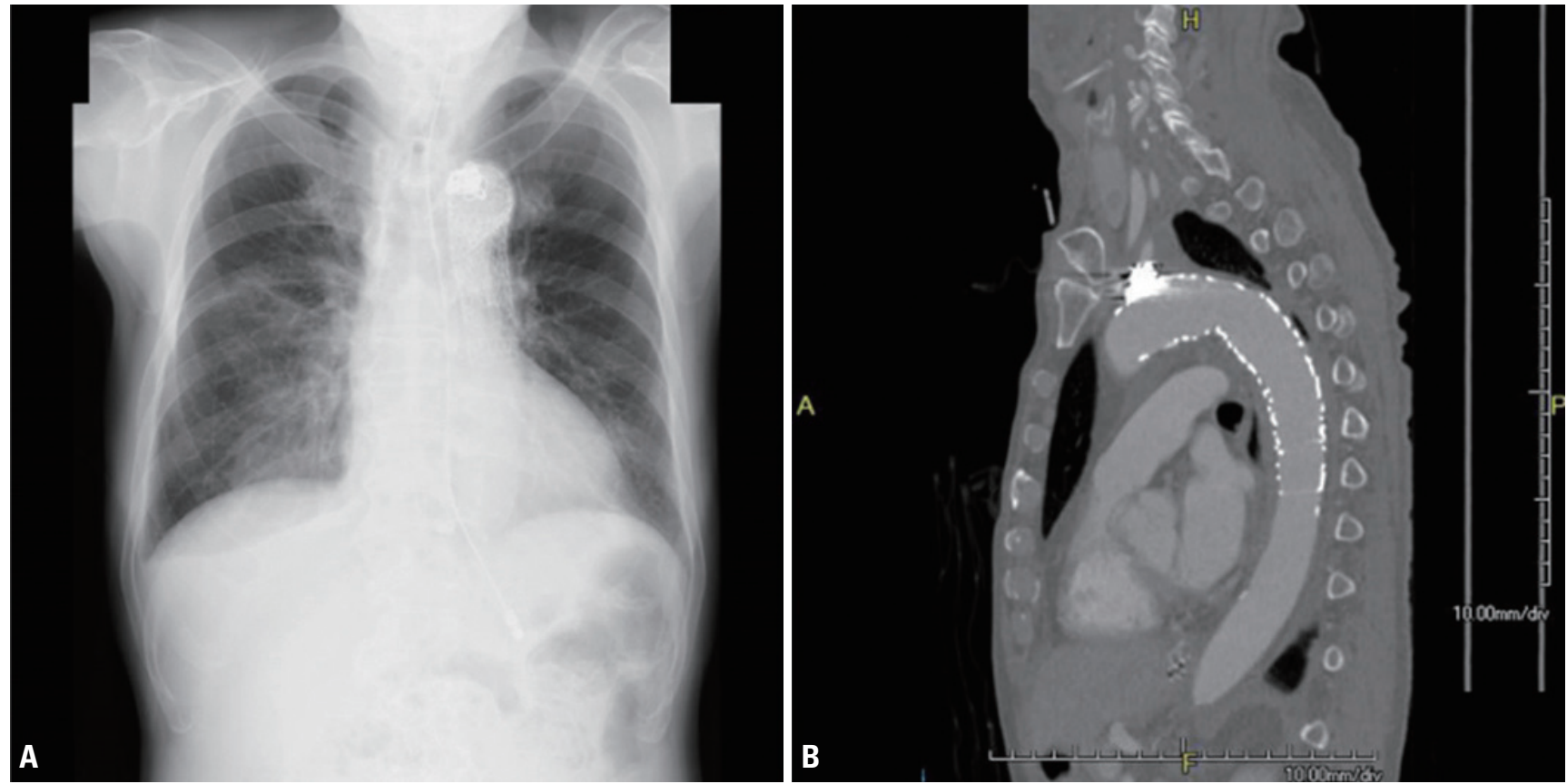

Fig. 2. The follow-up chest X-ray (A) and chest CT scan with contrast (B) on day 30. They did not show any complications related to TEVAR. CT: computerized tomography, TEVAR: thoracic endovascular aortic repair.

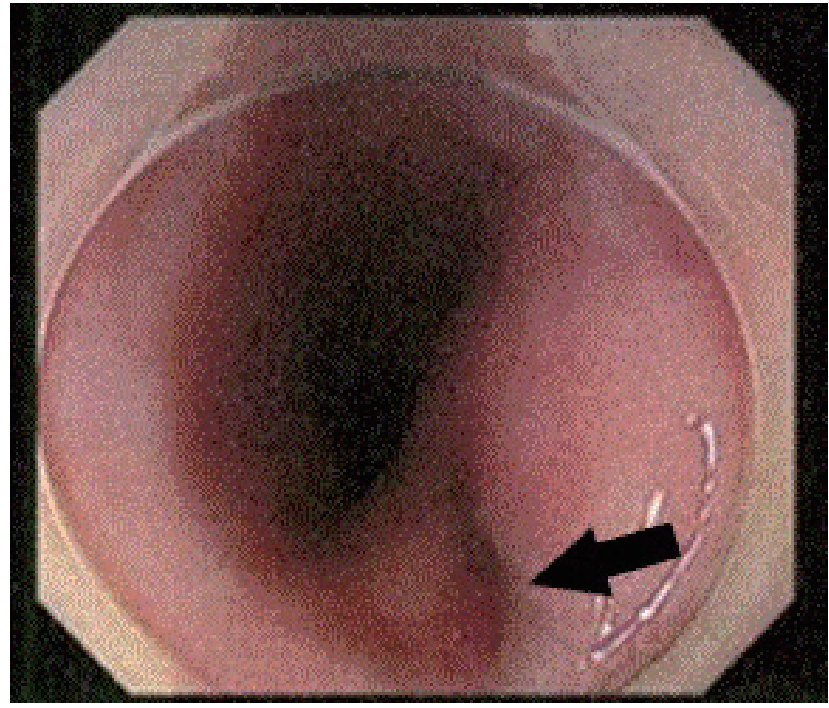

Fig. 3. The gastroscopy at the previous hospital. It revealed a cavity with clot suggesting AEF on the upper esophagus (black arrow). AEF: aorto-esophageal fistula.

\section{DISCUSSION}

Recently TEVAR has become one of the main therapies for aortic diseases, even if the cause was trauma [1-3].

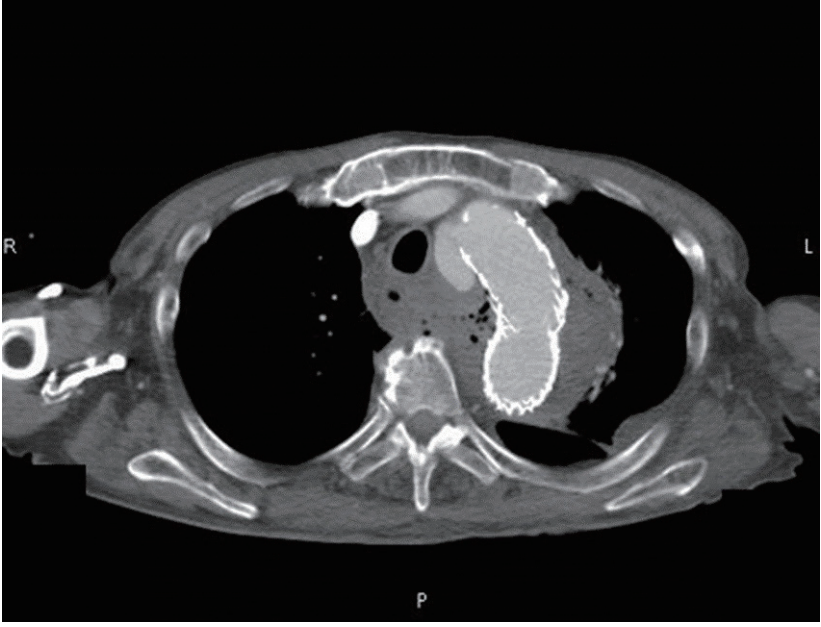

Fig. 4. The CT scan with contrast on the day of re-transfer to Niigata University Medical and Dental Hospital. A pseudoaneurysm of the aorta and free gas bubbles were in his mediastinum. These findings indicated AEF. CT: computerized tomography, AEF: aorto-esophageal fistula.

With regards to this case, we recognize that only TEVAR made it possible for him to survive his first hospitalization at Niigata University Medical and Dental Hospital because he wasn't seeming to tolerate the conventional open repair of the aorta due to serious conditions upon 
his arrival. This less invasive procedure must have provided him with a good postoperative course during his first hospitalization.

On the other hand, the long-term effects of TEVAR are still under investigation and there is no valid follow-up strategy after TEVAR in BTAI cases. According to current guidelines, the European Society of Cardiology recommends that follow-up should be performed at 1 month, 6 months, and 12 months after the treatment [4]. The European Society for Vascular Surgery requires CT angiography within 1 month and 12 months after the procedure [5]. But the quality of evidence for articles quoted in such guidelines remains low. We followed these guidelines in this case but could not predict the unfortunate ending.

$\mathrm{Xi}$ and colleagues reported that AEF developed in about $2 \%$ of patients undergoing TEVAR. It occurred between 1 month and 4 years after the procedure, most likely due to stent graft infection [6]. In our case, he developed AEF 3 months after TEVAR, although he had no symptoms during his 2-month hospitalization at Niigata University Medical and Dental Hospital.

Frailty is a geriatric syndrome characterized by vulnerability to stressors due to loss of physiologic reserve and has the potential to affect the prognosis of patients in clinical settings. Currently, this risk predictor has become an important modality for identifying high-risk patients undergoing major vascular surgery $[7,8]$. This case was 72 years old and might have suffered from alcohol use disorder. He was unable to walk without assistance despite 3 months of rehabilitation. He also seemed to be mentally drained. We accepted that he was frail. If we had recognized the frailty earlier and provided him with more strict follow-up for a longer time, early detection and treatment of AEF may have been possible.

In conclusion, we report a case of multiple trauma including BTAI with the lethal triad. He successfully underwent TEVAR but eventually died because of AEF. Fatal complications like AEF may occur after TEVAR, so clinicians need to carefully follow patients who underwent TEVAR.

\section{REFERENCES}

1. Ultee KH, Soden PA, Chien V, Bensley RP, Zettervall SL, Verhagen HJ, et al. National trends in utilization and outcome of thoracic endovascular aortic repair for traumatic thoracic aortic injuries. J Vasc Surg 2016;63:1232-9.

2. Patelis N, Katsargyris A, Klonaris C. Endovascular repair of traumatic isthmic ruptures: special concerns. Front Surg 2017;4:32.

3. Akhmerov A, DuBose J, Azizzadeh A. Blunt thoracic aortic injury: current therapies, outcomes, and challenges. Ann Vasc Dis 2019;12:1-5.

4. Erbel R, Aboyans V, Boileau C, Bossone E, Bartolomeo RD, Eggebrecht $\mathrm{H}$, et al. 2014 ESC guidelines on the diagnosis and treatment of aortic diseases: document covering acute and chronic aortic diseases of the thoracic and abdominal aorta of the adult. The task force for the diagnosis and treatment of aortic diseases of the European Society of Cardiology (ESC). Eur Heart J 2014;35:2873-926.

5. Riambau V, Böckler D, Brunkwall J, Cao P, Chiesa R, Coppi G, et al. Editor's choice - management of descending thoracic aorta diseases: clinical practice guidelines of the European Society for Vascular Surgery (ESVS). Eur J Vasc Endovasc Surg 2017;53:452.

6. Xi EP, Zhu J, Zhu SB, Zhang Y. Secondary aortoesophageal fistula after thoracic aortic aneurysm endovascular repair: literature review and new insights regarding the hypothesized mechanisms. Int J Clin Exp Med 2014;7:3244-52.

7. Gomibuchi T, Seto T, Komatsu M, Tanaka H, Ichimura H, Yamamoto T, et al. Impact of frailty on outcomes in acute type a aortic dissection. Ann Thorac Surg 2018;106:1349-55.

8. Furukawa H, Yamane N, Honda T, Yamasawa T, Kanaoka Y, Tanemoto K. Initial clinical evaluation of preoperative frailty in surgical patients with Stanford type A acute aortic dissection. Gen Thorac Cardiovasc Surg 2019;67:208-13. 